\title{
Cortical and cardiovascular responses to acute stressors and their relations with
} psychological distress

Agostino Brugnera $^{a \star}$, Cristina Zarbo ${ }^{a}$, Roberta Adornia ${ }^{a}$, Giorgio A. Tascab ${ }^{b}$ Massimo Rabbonic, Emi Bondic ${ }^{\mathrm{c}}$, Angelo Compare ${ }^{\mathrm{ad}}$, and Kaoru Sakatani ${ }^{\mathrm{e}}$

${ }^{a}$ Department of Human \& Social Sciences, University of Bergamo, Italy

${ }^{\mathrm{b}}$ School of Psychology, University of Ottawa, Canada

${ }^{c}$ Department of Psychiatry, Hospital Papa Giovanni XXIII, Bergamo, Italy

${ }^{d}$ Human Factors and Technology in Healthcare, University of Bergamo, Italy

e NEWCAT Research Institute, Department of Electrical and Electronic Engineering, College of Engineering, Nihon University, Japan

* Corresponding author

Correspondence:

Agostino Brugnera,

Department of Human \& Social Sciences, University of Bergamo, Piazzale S. Agostino 2, 24129 Bergamo, Italy agostino.brugnera@unibg.it 


\section{Abstract}

The purpose of this study was to assess the interrelation between cortical, cardiovascular, behavioural, and psychological responses to acute stressors in a large sample of healthy individuals. To date, there are only preliminary evidences for a significant association among these psychophysiological indexes during a stress task.

65 participants completed psychological questionnaires (Beck Depression Inventory and State-Trait Anxiety Inventory) and underwent a psychosocial math stress task, consisting of a control and an experimental (i.e. stressful) condition. Prefrontal and autonomic activity were recorded using respectively a 2-channel near-infrared spectroscopy (NIRS) device and a portable ECG monitoring system.

Results evidenced an increased activation of both frontal areas assessed by NIRS, and a positive association between the right NIRS channel and heart rate changes from baseline, during both control and experimental conditions. Subjective stress increased during the procedure, reaching its maximum during the experimental condition. Behavioural performances during the task (e.g. response time) did not correlate with anxiety or depression. Autonomic data evidenced, as expected, an overall reduction of vagal tone during the experimental condition. Finally, severity of depressive and anxious symptoms predicted an increase in parasympathetic activity both at rest and during the task, even when controlling for respiration rate.

Results support the hypothesis of an integration between right sectors of frontopolar or dorsolateral PFC and cardiac regulation. Trait anxiety and depression predicted an increase in vagal tone during the entire procedure. The implication of these findings is discussed. 


\section{INTRODUCTION}

Stress responses aim at maintaining the homeostasis of the organism in reaction to environmental stressors and preparing it to action (Carroll et al., 2012; Ulrich-Lai \& Herman, 2009). Nevertheless, prolonged stress responses could lead to adverse medical conditions (e.g. hypertension) through several physiological mechanisms (Segerstrom \& Miller, 2004). However, further studies are required to clarify the interrelation between physiological, behavioural and cognitive aspects of stress, which seem to be strictly related to state and trait psychological conditions.

From a general point of view, stress results in complex activationldeactivation patterns of various cerebral structures (Kogler et al., 2015) as well as to the activation of the Autonomic Nervous System (ANS) and of neuroendocrine systems, such as the hypothalamic-pituitaryadrenal axis (Ulrich-Lai \& Herman, 2009). Among cortical structures, Prefrontal Cortex (PFC) seems one of the most implicated in stress responses (Brugnera, Compare, \& Sakatani, 2016; Doi, Nishitani, \& Shinohara, 2013; Kern et al., 2008; Kogler et al., 2015; Takizawa, Nishimura, Yamasue, \& Kasai, 2014). Near-Infrared Spectroscopy (NIRS) is a recent non-invasive technology based on the properties of light; it allows assessing PFC activation patterns during a stress task in terms of concentration changes in cortical haemoglobin. Past NIRS studies suggested that stress is associated with an hyperactivation of right sectors of prefrontal cortex (Sakatani, Tanida, \& Katsuyama, 2010; Tanida, Katsuyama, \& Sakatani, 2007; Tanida, Sakatani, Takano, \& Tagai, 2004), even if some papers reported a general frontal activation (Takizawa et al., 2014; Yang et al., 2009).

It is well known that emotive, cognitive and affective information could be processed asymmetrically (Seghier, 2008). Indeed, differences among left and right hemispheres (e.g. during stress or affective tasks) has been widely reported in functional neuroimaging studies (Seghier, 2008): language, for example, is a widely recognized asymmetrical cognitive function (Benn, Zheng, Wilkinson, Siegal, \& Varley, 2012; Seghier, 2008). The hemispheric dominance 
could be investigated using a measure called Laterality Index (LI), that is the index of the left or right asymmetry of PFC activity. This measure is particularly suitable to investigate prefrontal asymmetries using 2-channel NIRS devices (Tanida et al., 2004). For example, Tanida and colleagues (2004) using a Laterality Index found that individuals with higher HR increases had a large activity in right prefrontal cortex than in the left one, during a stress task.

Right prefrontal cortex, according to the so-called "approach-withdrawal hypothesis" of emotions processing (Brugnera, Adorni, Compare, Zarbo, \& Sakatani, 2016), seems to have a key role in mediating withdrawal behaviours and negative emotions, as supposedly experienced during stress responses (Davidson, Ekman, Saron, Senulis, \& Friesen, 1990). This hypothesis is supported by evidence that higher state anxiety is associated with higher right PFC activation (Ishikawa et al., 2014; Takizawa et al., 2014). However, few NIRS studies have investigated the role of psychological distress (e.g. depression or trait anxiety) on prefrontal cortex activity during a stress task. These relations, considering the negative effect of psychological distress (in particular anxiety) on attentional control and working memory system (Attentional Control Theory; Eysenck, Derakshan, Santos, \& Calvo, 2007), deserve more attention. Preliminary evidence suggests that cognitive performance could also be reduced in individuals without psychological distress, in case of they show high stress responses or if they perceive the stressor as uncontrollable (Henderson, Snyder, Gupta, \& Banich, 2012).

Evidence suggests also a different hemispheric lateralization of systems that control the autonomic nervous system. Indeed, left hemisphere seems to regulate the parasympathetic activity of the autonomic nervous system (Wittling, Block, Genzel, \& Schweiger, 1998), while right hemisphere appears to be dominant in the control of the emotion processing (e.g. Heilman \& Gilmore, 1998) and the sympathetic activity (Critchley, Corfield, Chandler, Mathias, \& Dolan, 2000; Heilman, Schwartz, \& Watson, 1978; Wittling \& Genzel, 1995). For example, past NIRS studies showed that a right PFC activity was correlated with increased heart rates and parasympathetic withdrawal during stressful tasks (Sakatani et al., 2010; Tanida et al., 2007; 
Tanida et al., 2004). According to the model of neurovisceral integration (Thayer, Hansen, Saus-Rose, \& Johnsen, 2009; Thayer \& Lane, 2000), the central autonomic network (CAN) is a functionally integrated system through which specific brain areas (such as ventromedial PFC and amygdala) control visceromotor, neuroendocrine, and behavioural responses that are critical for goal-directed behaviours and adaptability (such as responses to stressful stimuli; Thayer et al., 2009). In addition to the main CAN areas, other anatomical regions selectively regulate one of the two branches of the autonomic nervous system, such as right dorsolateral PFC (Beissner, Meissner, Bar, \& Napadow, 2013). The final cerebral structure of the CAN is the brainstem, that directly regulates the heart rate as well the Heart Rate Variability (HRV; Thayer et al., 2009). HRV, the physiological phenomenon of variation in the beat-to-beat interval, is considered the principal index of central-peripheral neural feedback and of central-autonomic nervous system integration (Thayer \& Lane, 2000).Specific analyses of Heart Rate Variability can provide useful information about the cardiac ANS modulation: for example, frequency domain analyses of fixed frequency bands of HRV (i.e. High Frequencies, HF) offer a direct index of parasympathetic (or vagal) activity over the cardiac tone (Task Force of the European Society of Cardiology, 1996).

It is well known that cognitive tasks, increases in task difficulty and in attentional demand strongly suppress the parasympathetic influences on the heart, thus leading to increased HR, decreased HRV and lowered High Frequencies (Hansen, Johnsen, \& Thayer, 2003; Luft, Takase, \& Darby, 2009; Overbeek, van Boxtel, \& Westerink, 2014; Pendleton, Sakalik, Moore, \& Tomporowski, 2016). Moreover, a recent meta-analysis has evidenced that stress responses alter ANS, leading to sympathetic activation and parasympathetic withdrawal (Castaldo et al., 2015). Castaldo and colleagues (2015) concluded that during stress tasks high frequencies are significantly depressed.

High Frequencies seem also to be related to state and trait psychological conditions. High levels of psychological distress (i.e. state and trait anxiety; depression) are associated with reduced 
HRV both at rest and in response to stressors (Chalmers, Quintana, Abbott, \& Kemp, 2014; Kemp, Quintana, Felmingham, Matthews, \& Jelinek, 2012; Kemp et al., 2010). These results are important because a lower overall HRV at rest is considered a maladaptive stress response which could constitute a risk factor for increased adverse cardiac events (Porges, 2007). The association between psychological distress and reduced Heart Rate Variability can also be interpreted taking into account the above mentioned model of neurovisceral integration (Thayer \& Lane, 2000). Central autonomic network is impaired in distressed individuals and this impairment leads to a reduction of both parasympathetic tone and behavioural flexibility (Thayer et al., 2009). Indeed, distressed individuals are more likely to be worried and hypervigilant even when no real threat exists as well unable to disengage threat detection (Thayer et al., 2009). However, the debate about the link between psychological distress and stress responses is still open: few other studies have found, for example, a relationship between high levels of psychological distress and increased vagal tone or lower sympathetic activation during a stress task (Liang, Lee, Chen, \& Chang, 2015; Sanchez-Gonzalez et al., 2015; Shinba et al., 2008; Traina, Cataldo, Galullo, \& Russo, 2011).These results could be explained taking into account the conservation-withdrawal pattern, considered the counterpart of fight-flight reactions (Buerki \& Adler, 2005). To date, few studies found evidence of conservation-withdrawal pattern in humans (Bosch et al., 2001).

A critical aspect of the reviewed literature on responses to stress tasks, is the presence of a number of limitations: for example, NIRS literature is characterized by the lack of a control condition, the use of a single-gender sample, and usually small sample sizes.

This study aimed to address most of these gaps and clarify response patterns to psychosocial stress, integrating information from cortical (i.e. PFC), autonomic (i.e. HR and HRV), psychological (i.e. anxiety and depression) and behavioural data (i.e. performance to math task). In order to achieve these goals, we performed a study with a large sample of healthy individuals of both sexes, using a computerized and standardized procedure called Montreal 
Imaging Stress Task (MIST). MIST allows to administer both an experimental and a control condition, that are respectively with and without a social and time stressful pressures; during the procedure, individuals have to solve arithmetic operations. Cognitive performances could be collected on both conditions (Dedovic et al., 2005). Past literature has shown that MIST is a reliable protocol to induce physiological stress at CNS level (Kogler et al., 2015), even if ANS changes during this procedure have been underexamined. To the best of our knowledge, only 3 studies investigated vagal reactivity during this specific stress task, showing mixed results (Janka et al., 2015; La Marca et al., 2011; Monge, Gomez, \& Molina, 2014).

Therefore, in this study we tested the hypotheses that (i) the procedure will be effective and will induce significant changes in perceived stress (as assessed by manipulation checks); (ii) the experimental condition (i.e. stress task) will lead to increased heart rates and parasympathetic withdrawal, as supported by the meta-analysis of Castaldo and colleagues (2015); (iii) the experimental condition will lead to increased activity of right PFC, as partly supported by previous NIRS studies (Sakatani et al., 2010; Tanida et al., 2007); (iv) ANS and PFC activity will be positively and significantly correlated, as suggested by the model of neurovisceral integration (Beissner et al., 2013; Thayer et al., 2009; Thayer \& Lane, 2000); (v) psychological, cortical and performance outcomes will be significantly and positively correlated, as supported by the Attentional Control Theory (Eysenck et al., 2007) and previous studies (Takizawa et al., 2014); (vi) psychological distress (i.e. depression and trait anxiety) will have a negative predictive value on parasympathetic activity at rest, as supported by two previous meta-analyses (Chalmers et al., 2014; Kemp et al., 2010), and putatively during the entire procedure.

\section{MATERIAL AND METHODS}

\subsection{Participants}

A total of 65 healthy participants ( $52.3 \%$ females) with a mean age of 24.7 years ( $S D=3.9$ ) volunteered for the experiment. Participants were mostly university students (69.2\%). Mean BMI 
was $21.88(S D=1.99)$. All of them were right-handed, as assessed with the Italian version of Edinburgh Handedness Inventory (Salmaso \& Longoni, 1985). None of the participants was affected by neurological, psychiatric or other medical (e.g. cardiological) illnesses as assessed by means of a semi-structured interview.

The study was conducted in accordance with APA (1992) ethical standards for the treatment of human experimental volunteers; each participant provided written consent in compliance with the Declaration of Helsinki (2013).

\subsection{Measures and Instruments}

\subsubsection{Beck Depression Inventory II (BDI-II)}

The Beck Depression Inventory II (BDI-II; Beck, Steer, \& Brown, 1996; Ghisi, Flebus, Montano, Sanavio, \& Sica, 2006) is a self-report 21 -item measure of depressive symptoms used in a variety of settings and populations. It measures the rate of both psychological/mental and somatic manifestations of major depressive episodes during a 2-weeks period, as stated in the Diagnostic and Statistical Manual of Mental Disorder IV TR (DSM-IV TR; American Psychiatric Association, 1994). Each item is rated on a 4-point Likert scale (0 to 3). Total score ranges from 0 to 63 , with higher score reflecting higher symptoms severity.

BDI-II has demonstrated good consistency, sensitivity, validity, reliability and capacity to discriminate between depressed and non-depressed individuals (Wang \& Gorenstein, 2013). Internal consistency is around .9 , while the retest reliability and the convergent validity range respectively from .73 to 0.96 and from .82 to .94 (Wang \& Gorenstein, 2013). Good psychometric properties of the instrument were confirmed also in the Italian adaptation: internal consistency was .86, while convergent validity was .77 (Ghisi et al., 2006). Internal consistency in our sample was good $(\alpha=.86)$.

\subsubsection{State-Trait Anxiety Inventory (STAI)}

The State-Trait Anxiety Inventory (STAI; Pedrabissi \& Santinello, 1989; Spielberger, Gorsuch, Lushene, Vagg, \& Jacobs, 1983) is a self-report 40-item measure to assess both the presence 
and severity of usual propensity to be anxious (Trait scale) and current symptoms of anxiety (State scale). Each item is rated on a 4-point Likert scale (1 to 4). Total score for both scales ranges from 20 to 80 . Higher total scores represent higher anxiety severity.

STAI is a reliable and useful instrument to assess trait and state anxiety in a variety of contexts and populations (Julian, 2011): test-retest reliability and internal consistency alpha coefficients range varies respectively from .31 to .86 and from .86 to .95 across studies (Julian, 2011). Convergent and discriminant validity, test-retest reliability and internal consistency of the Italian adaptation of the instrument are good (Pedrabissi \& Santinello, 1989). As regards STAI-Trait, internal consistency in our sample was excellent $(\alpha=.91)$; as regards STAI-State, internal consistency was excellent at both baseline $(\alpha=.91)$ and experimental condition $(\alpha=.92)$.

\subsubsection{Stress Rating Questionnaire (SRQ) and Task Engagement}

The Stress Rating Questionnaire (E. J. Edwards, Edwards, \& Lyvers, 2015) is a self-report 5item questionnaire developed to assess changes in stress awareness (M. S. Edwards, Burt, \& Lipp, 2006). Current stress is rated in a 7-point Likert scale (1 to 7) on five bipolar dimensions, that are Calm to Nervous, Fearless to Fearful, Relaxed to Anxious, Unconcerned to Worried, and Comfortable to Tense. Total score ranges from 5 to 35 . Higher scores indicate higher selfreported state stress. An internally-adapted version of the original measure has been utilized in this study; internal consistency in our sample was good at both baseline $(\alpha=.87)$, control $(\alpha=$ $.89)$ and experimental $(\alpha=.88)$ conditions.

Task Engagement (TE) was assessed asking participants how much stressed they felt in that moment in a range from 0 to 10, where 0 indicates the lowest level of stress and 10 the highest.

\subsubsection{Montreal Imaging Stress Task (MIST)}

We used a within-subjects computerized protocol based on the "Montreal Imaging Stress Task" (MIST; Dedovic et al., 2005) to induce psychosocial stress in participants. The protocol had two test conditions (control and experimental). In the control condition, participants had to solve a series of simple arithmetic operations (sums and subtractions) displayed on the computer 
screen. In the experimental condition, the same type of arithmetic operations was proposed, but the participants had a time limit to solve them and a social pressure induced by the experimenter. The time limit of the operations was manipulated to be just beyond the individual's "mental capacity". Indeed, the experimental session was preceded by a 2 min training session; time recorded during the training session was used to set a default time limit for the experimental condition. During the experimental condition, after three correct responses the time limit decreased by $10 \%$; on the contrary, after three incorrect responses the time limit increased by $10 \%$ (see Dedovic et al., 2005 for the details of this procedure). MIST is an effective protocol, and has been extensively adopted in previous fMRI studies on stress responses (Kogler et al., 2015).

\subsubsection{NIRS Measurements}

We used a two channel CW-NIRS system (PocketNIRS Duo, DynaSense, Japan) for measurements of the relative concentration changes of oxy-haemoglobin $(\Delta \mathrm{Oxy}-\mathrm{Hb})$, deoxyhaemoglobin ( $\Delta$ deOxy-Hb), and total-haemoglobin $(=\Delta \mathrm{Oxy}-\mathrm{Hb}+\Delta \mathrm{deOxy}-\mathrm{Hb})$ in the PFC. This device employs a wireless communication system (Bluetooth®) and it uses light emitting diodes of three different wavelengths $(735 \mathrm{~nm} \pm 15,810 \mathrm{~nm} \pm 18,850 \mathrm{~nm} \pm 20)$ as light sources and one photo-diode as a detector: in each probe, the distance between emitter and detector is 3 $\mathrm{cm}$. The sampling rate was set to $10.1 \mathrm{~Hz}$. The concentration changes of haemoglobin were expressed in arbitrary units (a.u.).

The two optical probes were set symmetrically on the forehead with a flexible fixation pad, and were secured by an elastic band. The present montage replicated the one adopted by Tanida and colleagues (2007): this positioning is similar to the midpoint between electrode positions Fp1/Fp3 (left) and Fp2/F4 (right) of the international electroencephalographic 10-20 system (Tanida et al., 2007; see figure 2), with the emitters-detectors located over the dorsolateral and frontopolar areas of the PFC. 
The raw NIRS data were post-processed using a freely-available MATLAB toolbox (N.A.P., NIRS Analysis Package; Fekete, Rubin, Carlson, \& Mujica-Parodi, 2011) in order to correct periodic physiological (heart beats, respiration and blood pressure waves) and motion artefacts. Analyses were performed on both Oxy- and deOxy-Hb mean concentration changes from baseline.

\subsubsection{ECG Measurements}

Psychophysiological indexes were collected continuously throughout the protocol using "Pulse", a wearable device with a sampling rate of $256 \mathrm{~Hz}$, designed by STMicroelectronics and manufactured by MR\&D (Italy). The Pulse device continuously monitors heart's activity using an electric signal detection system. The device was fixed to the person's chest using an elastic band, which contained the electrodes. The data of each participant was visually inspected to detect and interpolate artefacts (i.e. missing or extra beats) using a piecewise cubic spline interpolation method (Tarvainen, Niskanen, Lipponen, Ranta-Aho, \& Karjalainen, 2014). Frequency domain (FD) indexes of Heart Rate Variability and mean heart rate (HR) were computed from raw ECG using a freely available MATLAB toolbox (Kubios HRV; Tarvainen et al., 2014). Regarding the FD analyses, data were pre-processed using a smoothness priors based detrending approach, which "operates like a time varying FIR high pass filter" (Tarvainen, Ranta-Aho, \& Karjalainen, 2002), thus removing very low components of HRV. The interbeat interval time series was interpolated with a rate of $4 \mathrm{~Hz}$ using a cubic spline interpolation to have equidistantly sampled data for spectral analysis. The power spectral density (PSD) was calculated by means of a Fast Fourier Transform (FFT) using the Welch's periodogram method, with a Hanning window width of 150 s and an overlap of $50 \%$. The main spectral component of interest, the High Frequency (HF), was evaluated in a fixed frequency band $(\mathrm{HF}=0.15-0.40$ $\mathrm{Hz}$ ); analyses were performed on absolute power values of HF (HFpow) calculated with FFT. An ECG-derived respiration rate (EDR) was computed for each condition in order to control for confounding role of respiration in some HFpow analyses. 


\subsection{Procedure}

Before the procedure participants completed psychological and manipulation-check questionnaires. After each condition, subjects completed manipulation-check questionnaires; STAI-State was administered only at baseline and after the experimental condition. During the procedure they were seated in a comfortable chair, in front of a computer, in a silent room with constant temperature. They were instructed to limit movements of the body and the head in order to reduce the amount of noise during psychophysiological recording. A 5 min rest period (baseline) was followed by the math task designed to induce moderate psychosocial stress (see figure 1). NIRS and ECG signals were collected during the entire procedure. The order of presentation of the control and experimental conditions was randomized across participants; each condition lasted 5 minutes. Mean baseline values of psychological questionnaires are reported in table 1.

\subsection{Statistical Analyses}

In order to test hypothesis 1 about the effectiveness of the procedure, behavioural data (i.e. response times and percentage of correct responses) and manipulation checks (i.e. TE, SRQ and STAI-State) scores were subjected to paired-sample t-tests. TE and SRQ scores were evaluated in terms of the difference from the mean baseline values, while STAI-State scores at baseline were compared to post-experimental ones. Independent variables were control and experimental condition.

In order to test hypothesis 2 that psychosocial stress would lead to an increase of HR and a reduction of HFpow, we used paired-sample t-tests. All dependent variables were evaluated in terms of the difference from the mean baseline values. Independent variables were control and experimental condition.

In order to test hypothesis 3 of a higher activation of right PFC during the experimental condition, we used a 2x2 Repeated Measures ANOVA (RMANOVA). Factors were Condition (2 levels: control, experimental) and NIRS Channels (2 levels: right, left). NIRS signal was 
analysed in terms of the difference from the mean baseline values; the dependent variables were Oxy- and deOxy-Hb concentration changes. Multiple Post-Hoc mean comparisons were performed using the Tukey's test.

In order to test hypothesis 4 of a significant correlation between ANS (HR, HF) and cortical indexes collected through NIRS (mean Oxy- and deOxy-Hb concentration changes, Laterality Indexes), we used Pearson's two-tailed correlation coefficients. We calculated the Laterality Index during baseline (LI-B), control (LI-C) and the experimental conditions (LI-E), following the equation proposed by Ishikawa and colleagues, for both Oxy- and deOxy-Hb (Ishikawa et al., 2014). LI provides values in the range of \pm 1 . A positive $\mathrm{LI}$ indicates that the right channel is, on average, more active than the left channel. On the contrary, a negative $\mathrm{LI}$ indicates that left channel is, on average, more active than the right one.

In order to test hypothesis 5 of significant correlations between psychological questionnaires (STAI and BDI) and both NIRS (Oxy- and deOxy-Hb concentration changes, Laterality Indexes) and behavioural data (\% of correct responses, number of correct responses, response times) during each condition, we used Pearson's two-tailed correlation coefficients.

Finally, in order to test hypothesis 6 of a significant correlation between psychological questionnaires (STAI and BDI) and HR as well as parasympathetic activity (HF) during each condition, we used Pearson's two-tailed correlation coefficients. Moreover, six hierarchical multiple linear regression analyses were used to assess the effect of depression (BDI) and anxiety (STAI-Trait) on HF during the three different conditions. We controlled for respiration rate (block 0) and entered the independent variables of interest (BDI or STAI-Trait) in block 1. No other control variables were inserted in block 0 due to the absence of significant correlations between demographic variables (i.e. age, sex, BMI) and HF, and the limited sample size (Tabachnick \& Fidell, 2007). 
All statistical analyses were performed using Statistical Package for the Social Sciences (SPSS) version 23.0 and STATISTICA version 12.5. All statistical tests were two-sided; a p value $\leq .05$ was considered significant.

\section{RESULTS}

3.1 Preliminary data screening. Preliminary analyses revealed no effects of task order on results, except of response times and number of correct responses during control condition. RTs were lower and number of correct responses were higher if the first task was the experimental one, probably due to a habituation effect.

3.1.1 Univariate outliers. Standardized scores and box plots were used to identify univariate outliers. Variables with values +/- $3.29 S D$ from the mean were considered outliers (Tabachnick \& Fidell, 2007): four outliers were identified for $\Delta \mathrm{Oxy}-\mathrm{Hb}$ variables during the entire procedure, while one outlier was identified for $\Delta$ deOxy- $\mathrm{Hb}$ variables and STAI-State at baseline. These values were brought into range (Tabachnick \& Fidell, 2007). As regards behavioural data, RTs exceeded $\pm 2 S D$ were excluded

3.1.2 Normality of Distributions. Normality was assessed for each variable by examining boxplots, stem and leaf plots, histograms, and skewness and kurtosis values. BDI variable was slightly positively skewed: a square root transformation corrected the non-normality and the resultant new variable (BDlsqrt) was used for analyses. HFpow variables during all conditions were moderately positively skewed: a log10 transformation corrected the non-normality, and the log-transformed variables were used for analyses.

3.1.3 Multivariate outliers, multicollinearity and singularity. Mahalonobis distance was used to assess multivariate outliers, while Multicollinearity and Singularity were assessed using Pearson correlation coefficients and tolerance statistics. Analyses were run to check for assumptions of multiple regression analyses. Results revealed neither multivariate outliers nor issues with multicollinearity and singularity. 
3.1.4 Normality, linearity, homoscedasticity and independence of residuals. Scatterplot of the standardised residuals were used to assess residuals' assumptions. Analyses were run to check for assumptions of multiple regression analyses. Results revealed no issues with normality, linearity, homoscedasticity and independence of residuals.

3.1.5 Missing data. STAI-State scores were missing in one subject at the baseline. TE and SRQ scores were missing in one subject at the experimental condition. Due to recording problems, ECG of one subject during the experimental condition was not recorded. Missing data were not imputed and were treated as missing.

3.2 Hypothesis 1. Effectiveness of stress procedure. Mean RTs were significantly higher during the control $(M=3.481 \mathrm{~s}$; $\mathrm{SD}=.658)$ than during the experimental condition $(\mathrm{M}=2.548$ s.; $\mathrm{SD}=.331), t(64)=14.73, p<.0001,95 \% \mathrm{Cl}[-1.06,-.807], \mathrm{d}=1.53$. Moreover, both number and percentage of correct responses were higher during the control condition ( $\mathrm{N}$ Correct: $\mathrm{M}=$ 67.45; $S D=12.77$; Percentage of Correct: $M=96.56$; $S D=3.19$ ) than during experimental condition ( $N$ Correct: $M=47.08$; $S D=5.34$; Percentage of Correct: $M=52.01$; $S D=2.6$ ). The differences between means of both behavioral data were statistically significant: Number of correct responses, $t(64)=16.4, p<.0001,95 \% \mathrm{Cl}[-1.06,-.807], d=1.65 ;$ Percentage of correct responses, $t(64)=93.57 ; p<.0001,95 \% \mathrm{Cl}[-22.8,-17.9], d=15.6$. Therefore, behavioural results confirmed that the procedure worked as expected. As regards the Manipulation Check data, $\triangle T E$ increased significantly from control to experimental condition ( $\triangle \mathrm{TE}$ control: $\mathrm{M}=1.366 ; \mathrm{SD}=1.713 ; \Delta \mathrm{TE}$ experimental: $\mathrm{M}=2.031 ; \mathrm{SD}$ $=2.204), t(63)=-9.422, \mathrm{p}<.0001,95 \% \mathrm{Cl}[1.6,2.43], \mathrm{d}=-.98$, as well as $\triangle S R Q$ scores $(\triangle S R Q$ control: $M=-.246 ; S D=5.668 ; \Delta S R Q$ experimental: $M=4.75 ; S D=6.251), t(63)=-$ 8.633; $p<.0001,95 \% \mathrm{Cl}[3.94,6.31], d=-.87$. Finally, STAI-State scores increased significantly from baseline to experimental condition (STAI-State baseline: $\mathrm{M}=34.56$; SD = 7.06; STAI-State experimental: $\mathrm{M}=43.95 ; \mathrm{SD}=10.98), t(63)=-7.136, \mathrm{p}<.0001,95 \% \mathrm{Cl}[6.86,12], \mathrm{d}=-.96$. 
Results evidenced that procedure was associated with an increased perceived stress and anxiety (see figure 2).

3.3 Hypothesis 2. Stress and cardiovascular responses. $\Delta \mathrm{HR}$ increased as respect to the baseline during both control $(M=2.389 ; S D=4.682)$ and experimental conditions $(M=8.677$; $\mathrm{SD}=8.85)$. The two conditions were significantly different, $t(63)=-6.391, p<.0001,95 \% \mathrm{Cl}[-$ $8.25,-4.32], d=-.82$, evidencing that the stress procedure successfully increased HR. $\Delta \operatorname{logHFpow}$ decreased as respect to the baseline during both control $(\mathrm{M}=-.171 ; \mathrm{SD}=.309)$ and experimental conditions $(M=-.375 ; S D=.518)$. Moreover, the two conditions were significantly different, $t(63)=4.154 ; p<.0001,95 \% \mathrm{Cl}[.106, .302], \mathrm{d}=.43$. Thus, the stress procedure was associated with an overall reduction of vagal tone (see figure 3).

\subsection{Hypothesis 3. Stress and PFC responses. RMANOVA performed on Oxy-Hb}

concentration changes from baseline evidenced an effect of the Condition, $F(1,64)=25.19 ; p<$ .0001 , partial $\eta^{2}=.282$, with an overall increase of PFC activity in the area illuminated by both left and right channels during the experimental condition, as respect to the control one (Control: $\mathrm{M}=.021$ a.u., SE $=.005$; Experimental: $\mathrm{M}=.036$ a.u., SE $=.005)$. The effects of Channel $(p=$ $.5)$ and the interaction between Condition * Channel $(p=.94)$ were not significant.

Results on deOxy-Hb concentration changes from baseline evidenced a significant effect of the Condition, $F(1,64)=11.52 ; p=.005 ;$ partial $\eta^{2}=.51$, with an overall decrease of deoxygenated haemoglobin in the area illuminated by both left and right channels during the experimental condition as respect to the control one (Control: $\mathrm{M}=-.004, \mathrm{SE}=.0002$; Experimental: $\mathrm{M}=-.01$, $\mathrm{SE}=.003)$. There was neither a significant effect of Channel $(p=.398)$ nor of Condition * Channel $(p=.471)$.

Therefore, results evidenced an increase in bilateral PFC activity and no noticeable asymmetries during the experimental condition. A grand mean of the $\mathrm{Oxy}-\mathrm{Hb}$ and deOxy-Hb signal during the entire procedure is showed in figure 4. 
3.5 Hypothesis 4. Interrelationship between cortical and ANS indexes. $\Delta H R$ at both control and experimental condition positively correlated respectively with mean Oxy-Hb concentration changes in the right channel during control $(r=.291 ; p<0.001)$ and experimental $(r=.256 ; p=$ 0.041) conditions. Results evidenced that individuals with higher increases of HR from baseline, during both conditions experienced a higher NIRS' right channel activity. No significant correlations were found between other ANS and cortical indexes.

\subsection{Hypothesis 5. Psychological distress's relationship with cortical and behavioural} indexes. BDIsqrt, STAI-State and -Trait were all positively and significantly correlated between them (see table 2). No significant correlations were found between BDIsqrt or STAI-Trait and Oxy or Deoxy LI-B, LI-C, LI-E, any Oxy- or deOxy-Hb mean value or behavioural data. STAIState did not correlate with any physiological or behavioural variable. LI-E was negatively correlated with number of correct responses during experimental condition $(r=-.287 ; p=.02)$, showing that during the experimental condition individuals with increased NIRS' left channel activity gave a higher number of correct responses.

3.7 Hypothesis 6. Psychological distress and parasympathetic activity. As evidenced in table 2, an increase in BDIsqrt scores was significantly correlated with an increase in HR and logHFpow during all conditions, while STAI-Trait correlated significantly and positively only with logHFpow during all conditions.

The hierarchical multiple regression on logHFpow at baseline condition revealed that at Block 0 , EDR contributed significantly to the regression model ( $F$ change $1,63=5.258 ; p=.025)$ and accounted for $7.7 \%$ of the variation in logHFpow. Introducing the BDIsqrt explained an additional $7.9 \%$ of variation in logHFpow and this change in $\mathrm{R}^{2}$ was significant $(F$ change $2,62=$ $5.809 ; p=.019)$. The standardized beta value of BDlsqrt was .284 $(p=.019)$.

The regression on logHFpow at control condition revealed that EDR contributed significantly to model at Block 0 ( $F$ change $1,63=17.1 ; p<.001)$ and accounted for $21.3 \%$ of the variation in logHFpow. Introducing the BDIsqrt explained an additional $5.9 \%$ of variation in logHFpow and 
this change in $\mathrm{R}^{2}$ was significant $(F$ change 2,62 $=4.996 ; p=.029)$. The standardized beta value of BDlsqrt was .248 $(p=.029)$.

Finally, the regression on logHFpow at experimental condition revealed that EDR contributed significantly to model at Block 0 ( $F$ change $1,62=20.04 ; p<.001)$ and accounted for $24.4 \%$ of the variation in logHFpow. Introducing the BDIsqrt explained an additional $6.7 \%$ of variation in logHFpow and this change in $\mathrm{R}^{2}$ was significant $(F$ change $2,61=5.974 ; p=.017)$. The standardized beta value of BDIsqrt was $.262(p=.017)$. When entering the STAI-Trait scores at Block 1 instead of BDIsqrt, all results were still significant. STAI-Trait explained $5.9 \%$ of variance of logHFpow at baseline $(F$ change $2,62=4.211 ; p=.044), 5 \%$ at control $(F$ change $2,62=4.248 ; p=.043)$ and $6.3 \%$ at experimental condition $(F$ change $2,62=5.554 ; p=.022)$. Standardized beta scores for STAI-Trait were .244 $(p=.044)$ at baseline, .225 at control $(p=$ $.043)$ and .251 at experimental condition $(p=.022)$.

Regression analyses evidenced that higher STAI-Trait and BDIsqrt scores predicted an increase in parasympathetic activity during the entire procedure, even when controlling for respiration rate.

\section{DISCUSSION}

In summary, our results evidenced that stress response led to an activation of both prefrontal areas illuminated by NIRS, increased HR, parasympathetic withdrawal, and increased selfreported stress and anxiety levels. No lateralization effects on the PFC were found.

Interestingly, trait anxiety and depression predicted a contrasting increase in vagal activity both at rest and during the experimental procedure, suggesting altered cardiac responses in individuals with high levels of psychological distress.

Behavioural and manipulation checks data evidenced that procedure was effective and induced a significant amount of perceived stress. As regards behavioural data, response times, percentage of correct responses and total number of correct responses decreased from control to the time-limited experimental condition, due to protocol's characteristics (Dedovic et al., 
2005). Results evidenced an increase in perceived stress changes (evaluated through Task Engagement and Stress Rating Questionnaire) from control to experimental condition, while anxiety (evaluated through STAI-State) increased from baseline to experimental condition. As regards autonomic data, $\mathrm{HR}$ and logHF changes from baseline respectively increased and decreased from control to experimental condition. Thus, stress was associated with a parasympathetic withdrawal that led to higher heart rates and lower High Frequencies of Heart Rate Variability, in accordance with the meta-analysis of Castaldo and colleagues (Castaldo et al., 2015). Interestingly, of the 12 studies included in the aforementioned paper only 4 used an arithmetic task, among which no-one implemented a control condition (Castaldo et al., 2015). Our results clearly show that an arithmetic task is associated per-se with a stress-induced vagal withdrawal, which tends to decrease even more during a properly defined condition of psychosocial stress. Indeed, Delta logHF were negative even during the control condition. Moreover, our results expand previous ANS findings using the MIST protocol: to date, only two studies evidenced a similar parasympathetic withdrawal during the experimental condition of MIST (Janka et al., 2015; La Marca et al., 2011), while one showed no significant decreases (Monge et al., 2014).

As regards NIRS data, there was a significant increase of oxy-haemoglobin and a decrease of deoxy-haemoglobin in the regional brain tissue illuminated by Near-Infrared Spectroscopy (part of the frontopolar and dorsolateral PFC), during both conditions of the stress task. Therefore, in our sample the hypothesis of an asymmetrical activation of right prefrontal cortex due to the processing of acute stressors was not confirmed. It's worth noticing that the procedure induced significant perceived stress, as evidenced by manipulation checks, and that ANS data showed a stress-induced vagal inhibition. Other NIRS studies did not evidence a clear lateralization pattern in the PFC during a mental arithmetic task (Ishikawa et al., 2014; Yang et al., 2009). Sakatani, Tanida and colleagues $(2010 ; 2007 ; 2004)$ described a positive correlation from between right-shifted laterality indexes and autonomic (e.g. HR) indexes during a math task, 
inferring from these results that right prefrontal cortex is implied in stress responses. However, these studies were limited by the assumption that stress could be elicited simply by an arithmetic task, that could be roughly comparable to MIST control condition. MIST is a wellknown protocol to induce psychosocial stress: a recent meta-analysis on fMRI studies evidenced an increased activity of a right area of prefrontal cortex (pars triangularis of the right inferior frontal gyrus) during the experimental condition of MIST (Kogler et al., 2015). The activity of inferior frontal gyrus cannot be directly evaluated through a 2-channel NIRS device, whose optodes are typically positioned over dorsolateral and frontopolar areas, putatively explaining the lack of results.

Correlations among Heart Rate changes from baseline and $\mathrm{Oxy}-\mathrm{Hb}$ concentration changes in the right PFC were positive and significant during both control and experimental conditions. Results were consistent with previous NIRS studies (Sakatani et al., 2010; Tanida et al., 2007; Tanida et al., 2004), and support the hypothesis of an involvement of right dorsolateral and frontopolar PFC in regulating sympathetic responses during a stress task. According to the neurovisceral integration model specific regions (i.e. ventromedial prefrontal cortex, midcingulate cortex, left amygdala and insula) control both branches of ANS (Beissner et al., 2013). However, some brain areas, like dorsolateral prefrontal cortex, are associated only with sympathetic regulation, (dIPFC; Beissner et al., 2013); therefore, we speculate that our NIRS device permitted to evaluate the activity of the latter PFC region.

Correlational analyses evidenced a negative and significant association between Laterality Index and number of correct responses during experimental condition: therefore, individuals with increased left prefrontal cortex activity gave a higher number of correct responses. Neuroanatomical models of number processing postulate that "exact calculations involve language areas, as well as subcortical structures, mainly in the left hemisphere" (Benn, Zheng, Wilkinson, Siegal, \& Varley, 2012). During experimental condition participants were forced to quickly solve arithmetic tasks; thus, we hypothesize that those who gave a higher number of 
correct responses, were able to better recruit left hemisphere. No significant correlations were found between psychological questionnaires (BDI and STAI) and behavioural data. Therefore, the hypothesis of impaired performances in anxious individuals during a stress task (Attentional Control Theory; Eysenck et al., 2007) was not confirmed. It should be noted that the sample was composed by young healthy adults, whose behavioural responses were quite similar (standard deviations were small), reducing the probability to show a hypothetical low effect. In the same way, we did not find a significant correlation between psychological distress and cortical indexes, thus not confirming previous results on a relationship between anxiety (state or trait) and right PFC activity (Takizawa et al., 2014).

Finally, we performed hierarchical multiple regression analyses in order to examine the predictive value of trait anxiety and depression on High Frequencies of Heart Rate Variability, controlling for respiration rate. It is worth noticing that questionnaires used to evaluate anxiety and depression (STAI-Trait and BDI, respectively) were highly correlated between each other (see table 2); thus, they were probably referring to an underlying dimension that could be defined "psychological distress" (Burns \& Eidelson, 1998). Results of regression analyses evidenced that a higher psychological distress predicts an increase in parasympathetic activity at rest and during a stress task. These findings are consistent with those of correlational analyses (see table 2) and are opposite to our initial hypothesis. Indeed, two past metaanalyses evidenced that patients with depressive and anxiety disorders are characterized by lowered parasympathetic activity at rest (Chalmers et al., 2014; Kemp et al., 2010). However, both meta-analyses were conducted on articles including patients with psychiatric disorders, while in our study we recruited healthy individuals. To the best of our knowledge, this is the first study that clearly demonstrates an increased parasympathetic activity at rest in individuals with high levels of psychological distress. As regards parasympathetic activity during the stress task, our results are partly consistent with previous literature (Liang et al., 2015; Sanchez-Gonzalez et al., 2015; Shinba et al., 2008; Traina et al., 2011). In addition to these results, our study 
suggests that psychological distress predicts increased parasympathetic activity during an arithmetic task (i.e. control condition), as well as during a stress condition. A possible explanation takes into account desensitization processes: indeed, in healthy individuals anxiety leads to a progressive $\beta$-adrenergic receptor desensitization (Yu, Kang, Ziegler, Mills, \& Dimsdale, 2008). Therefore, we speculate that psychological distress could be associated to a chronic hyper-activation of sympathetic nervous system; thus, the excessive catecholaminergic stimulation reduces the sensitivity of $\beta$-adrenergic receptors, leading to an increased vagal tone both at rest and during a stress task. Another explanation takes into account the so-called conservation-withdrawal reaction pattern of disengagement (Kreibig, Wilhelm, Roth, \& Gross, 2007), which is often referred to as aversive coping style (Bosch et al., 2001). Conservationwithdrawal is a biological reaction pattern, characterized by an enhanced parasympathetic tone (Kreibig et al., 2007). We hypothesize that individuals with high levels of psychological distress adopted an aversive coping style before and during a stress task, leading to the observed increased parasympathetic activity. However, the association between Central Autonomic Network, conservation-withdrawal reaction pattern and the desensitization hypothesis is still unclear.

Our results were partly limited by the fact that we did not measure free cortisol levels in plasma or saliva: indeed, it is well-known that psychological stressors activate HPA axis, leading to an increase in cortisol concentrations (Dickerson \& Kemeny, 2004; Ulrich-Lai \& Herman, 2009). Thus, measuring free levels of this hormone could have provided a better evaluation of the physiological stress response. In addition, it is worth noticing that this response is influenced by (i) progesterone and the hormonal state during menstrual cycle in women (Woods, Lentz, Mitchell, \& Kogan, 1994), and by (ii) aerobic training among healthy adults (Zschucke, Renneberg, Dimeo, Wustenberg, \& Strohle, 2015); thus, future studies should evaluate stress responses controlling for these variables. 
Concluding, we performed a large, randomized and controlled study on stress responses, using both NIRS and ECG devices. Further multi-channel NIRS studies are required to investigate activity of specific areas of prefrontal cortex using the same task. Moreover, we found evidence of attenuated vagal withdrawal in individuals with high levels of psychological distress, both at rest and during the experimental procedure. However, we did not investigate other linear and non-linear indexes of $\mathrm{HRV}$; therefore, a comprehensive picture of cardiovascular activity during this procedure is still lacking. Future studies should investigate the role of specific coping styles during stress responses, the impact of conservation-withdrawal reaction patterns on health outcomes as well as other linear and non-linear indexes of HRV during this specific acute stress task. 


\section{REFERENCES}

American Psychiatric Association. (1994). Diagnostic and statistical manual of mental disorders DSM-IV-TR. Washington: American Psychiatric Publishing.

American Psychological Association. (1992). Ethical principles of psychologists and code of conduct. American psychologist, 47, 1597-1411. doi:10.1037/0003-066X.47.12.1597

Beck, A. T., Steer, R. A., \& Brown, G. K. (1996). BDI-II: Beck Depression Inventory Manual. San Antonio: Psychological Corporation.

Beissner, F., Meissner, K., Bar, K. J., \& Napadow, V. (2013). The autonomic brain: an activation likelihood estimation meta-analysis for central processing of autonomic function. $J$ Neurosci, 33(25), 10503-10511. doi:10.1523/JNEUROSCI.1103-13.2013

Benn, Y., Zheng, Y., Wilkinson, I. D., Siegal, M., \& Varley, R. (2012). Language in calculation: a core mechanism? Neuropsychologia, 50(1), 1-10. doi:10.1016/j.neuropsychologia.2011.09.045

Bosch, J. A., de Geus, E. J., Kelder, A., Veerman, E. C., Hoogstraten, J., \& Amerongen, A. V. (2001). Differential effects of active versus passive coping on secretory immunity. Psychophysiology, 38(5), 836-846. doi:10.1111/1469-8986.3850836

Brugnera, A., Adorni, R., Compare, A., Zarbo, C., \& Sakatani, K. (2016). Cortical and Autonomic Patterns of Emotion Experiencing During a Recall Task. J Psychophysiol. doi:10.1027/0269-8803/a000183

Brugnera, A., Compare, A., \& Sakatani, K. (2016). Assessment of Physiological Patterns of Acute Stress Responses: The Role of Near-Infrared Spectroscopy. In A. Compare, C. Elia, A. G. Simonelli, \& F. Cattafi (Eds.), Psychological Distress: Risk Factors, Patterns and Coping Strategies (pp. 167-180). Hauppauge NY Nova Science Publishers.

Buerki, S., \& Adler, R. H. (2005). Negative affect states and cardiovascular disorders: a review and the proposal of a unifying biopsychosocial concept. Gen Hosp Psychiatry, 27(3), 180188. doi:10.1016/j.genhosppsych.2004.12.003

Burns, D. D., \& Eidelson, R. J. (1998). Why are depression and anxiety correlated? A test of the tripartite model. J Consult Clin Psychol, 66(3), 461-473. doi:http://dx.doi.org/10.1037/0022-006X.66.3.461 
Carroll, D., Ginty, A. T., Der, G., Hunt, K., Benzeval, M., \& Phillips, A. C. (2012). Increased blood pressure reactions to acute mental stress are associated with 16-year cardiovascular disease mortality. Psychophysiology, 49(10), 1444-1448. doi:10.1111/j.14698986.2012.01463.x

Castaldo, R., Melillo, P., Bracale, U., Caserta, M., Triassi, M., \& Pecchia, L. (2015). Acute mental stress assessment via short term HRV analysis in healthy adults: A systematic review with meta-analysis. Biomedical Signal Processing and Control, 18, 370-377. doi:http://dx.doi.org/10.1016/i.bspc.2015.02.012

Chalmers, J. A., Quintana, D. S., Abbott, M. J., \& Kemp, A. H. (2014). Anxiety Disorders are Associated with Reduced Heart Rate Variability: A Meta-Analysis. Front Psychiatry, 5, 80. doi:10.3389/fpsyt.2014.00080

Critchley, H. D., Corfield, D. R., Chandler, M. P., Mathias, C. J., \& Dolan, R. J. (2000). Cerebral correlates of autonomic cardiovascular arousal: a functional neuroimaging investigation in humans. J Physiol, 523, 259-270. doi:10.1111/j.1469-7793.2000.t01-1-00259.x

Davidson, R. J., Ekman, P., Saron, C. D., Senulis, J. A., \& Friesen, W. V. (1990). Approachwithdrawal and cerebral asymmetry: emotional expression and brain physiology. I. J Pers Soc Psychol, 58(2), 330-341. doi:http://dx.doi.org/10.1037/0022-3514.58.2.330

Dedovic, K., Renwick, R., Mahani, N. K., Engert, V., Lupien, S. J., \& Pruessner, J. C. (2005). The Montreal Imaging Stress Task: using functional imaging to investigate the effects of perceiving and processing psychosocial stress in the human brain. J Psychiatry Neurosci, $30(5), 319-325$.

Dickerson, S. S., \& Kemeny, M. E. (2004). Acute stressors and cortisol responses: a theoretical integration and synthesis of laboratory research. Psychol Bull, 130(3), 355-391. doi:10.1037/0033-2909.130.3.355

Doi, H., Nishitani, S., \& Shinohara, K. (2013). NIRS as a tool for assaying emotional function in the prefrontal cortex. Front Hum Neurosci, 7, 770. doi:10.3389/fnhum.2013.00770

Edwards, E. J., Edwards, M. S., \& Lyvers, M. (2015). Cognitive trait anxiety, situational stress, and mental effort predict shifting efficiency: Implications for attentional control theory. Emotion, 15(3), 350-359. doi:10.1037/emo0000051 
Edwards, M. S., Burt, J. S., \& Lipp, O. V. (2006). Selective processing of masked and unmasked verbal threat material in anxiety: Influence of an immediate acute stressor. Cogn Emot, 20(6), 812-835. doi:http://dx.doi.org/10.1080/02699930500375761

Eysenck, M. W., Derakshan, N., Santos, R., \& Calvo, M. G. (2007). Anxiety and cognitive performance: attentional control theory. Emotion, 7(2), 336-353. doi:10.1037/15283542.7.2.336

Fekete, T., Rubin, D., Carlson, J. M., \& Mujica-Parodi, L. R. (2011). The NIRS Analysis Package: noise reduction and statistical inference. PLOS ONE, 6(9), e24322. doi:10.1371/journal.pone.0024322

Ghisi, M., Flebus, G. B., Montano, A., Sanavio, E., \& Sica, C. (2006). Manuale del BDI-II Beck Depression Inventory-II, Adattamento italiano [BDI-II, Beck Depression Inventory-II Manual, Italian adaptation]. Firenze, Italy: Giunti OS.

Hansen, A. L., Johnsen, B. H., \& Thayer, J. F. (2003). Vagal influence on working memory and attention. Int J Psychophysiol, 48(3), 263-274. doi:http://dx.doi.org/10.1016/S01678760(03)00073-4

Heilman, K. M., \& Gilmore, R. L. (1998). Cortical influences in emotion. J Clin Neurophysiol, 15(5), 409-423. doi:http://dx.doi.org/10.1097/00004691-199809000-00005

Heilman, K. M., Schwartz, H. D., \& Watson, R. T. (1978). Hypoarousal in patients with the neglect syndrome and emotional indifference. Neurology, 28(3), 229-232. doi:http://dx.doi.org/10.1212/WNL.28.3.229

Henderson, R. K., Snyder, H. R., Gupta, T., \& Banich, M. T. (2012). When does stress help or harm? The effects of stress controllability and subjective stress response on stroop performance. Front Psychol, 3, 179. doi:10.3389/fpsyg.2012.00179

Ishikawa, W., Sato, M., Fukuda, Y., Matsumoto, T., Takemura, N., \& Sakatani, K. (2014). Correlation between asymmetry of spontaneous oscillation of hemodynamic changes in the prefrontal cortex and anxiety levels: a near-infrared spectroscopy study. $J$ Biomed Opt, 19(2), 027005. doi:10.1117/1.JBO.19.2.027005 
Janka, A., Adler, C., Fischer, L., Perakakis, P., Guerra, P., \& Duschek, S. (2015). Stress in crisis managers: evidence from self-report and psychophysiological assessments. J Behav Med, 38(6), 970-983. doi:10.1007/s10865-015-9654-1

Julian, L. J. (2011). Measures of anxiety: State-Trait Anxiety Inventory (STAI), Beck Anxiety Inventory (BAI), and Hospital Anxiety and Depression Scale-Anxiety (HADS-A). Arthritis Care Res (Hoboken), 63 Suppl 11, S467-472. doi:10.1002/acr.20561

Kemp, A. H., Quintana, D. S., Felmingham, K. L., Matthews, S., \& Jelinek, H. F. (2012). Depression, comorbid anxiety disorders, and heart rate variability in physically healthy, unmedicated patients: implications for cardiovascular risk. PLoS One, 7(2), e30777. doi:10.1371/journal.pone.0030777

Kemp, A. H., Quintana, D. S., Gray, M. A., Felmingham, K. L., Brown, K., \& Gatt, J. M. (2010). Impact of depression and antidepressant treatment on heart rate variability: a review and meta-analysis. Biol Psychiatry, 67(11), 1067-1074. doi:10.1016/j.biopsych.2009.12.012

Kern, S., Oakes, T. R., Stone, C. K., McAuliff, E. M., Kirschbaum, C., \& Davidson, R. J. (2008). Glucose metabolic changes in the prefrontal cortex are associated with HPA axis response to a psychosocial stressor. Psychoneuroendocrinology, 33(4), 517-529. doi:10.1016/j.psyneuen.2008.01.010

Kogler, L., Muller, V. I., Chang, A., Eickhoff, S. B., Fox, P. T., Gur, R. C., \& Derntl, B. (2015). Psychosocial versus physiological stress - Meta-analyses on deactivations and activations of the neural correlates of stress reactions. Neuroimage, 119, 235-251. doi:10.1016/j.neuroimage.2015.06.059

Kreibig, S. D., Wilhelm, F. H., Roth, W. T., \& Gross, J. J. (2007). Cardiovascular, electrodermal, and respiratory response patterns to fear- and sadness-inducing films. Psychophysiology, 44(5), 787-806. doi:10.1111/j.1469-8986.2007.00550.x

La Marca, R., Waldvogel, P., Thörn, H., Tripod, M., Wirtz, P. H., Pruessner, J. C., \& Ehlert, U. (2011). Association between Cold Face Test-induced vagal inhibition and cortisol response to acute stress. Psychophysiology, 48(3), 420-429. doi:http://dx.doi.org/10.1111/j.1469-8986.2010.01078.x 
Liang, C. S., Lee, J. F., Chen, C. C., \& Chang, Y. C. (2015). Reactive heart rate variability in male patients with first-episode major depressive disorder. Prog Neuropsychopharmacol Biol Psychiatry, 56, 52-57. doi:10.1016/j.pnpbp.2014.08.004

Luft, C. D., Takase, E., \& Darby, D. (2009). Heart rate variability and cognitive function: effects of physical effort. Biol Psychol, 82(2), 164-168. doi:10.1016/j.biopsycho.2009.07.007

Monge, M. M., Gomez, I., \& Molina, A. (2014). Robustness of Parameters from Heart Rate for Mental Stress Detection. Exp Clin Cardiol, 20(8), 3509-3517.

Overbeek, T. J., van Boxtel, A., \& Westerink, J. H. (2014). Respiratory sinus arrhythmia responses to cognitive tasks: effects of task factors and RSA indices. Biol Psychol, 99(1), 1-14. doi:10.1016/j.biopsycho.2014.02.006

Pedrabissi, L., \& Santinello, M. (1989). Inventario per l'ansia di «Stato» e di «Tratto»: nuova versione italiana dello STAI Forma Y: Manuale. Firenze, Italy: Giunti OS.

Pendleton, D. M., Sakalik, M. L., Moore, M. L., \& Tomporowski, P. D. (2016). Mental engagement during cognitive and psychomotor tasks: Effects of task type, processing demands, and practice. Int J Psychophysiol. doi:10.1016/j.ijpsycho.2016.08.012

Porges, S. W. (2007). The polyvagal perspective. Biol Psychol, 74(2), 116-143. doi:10.1016/j.biopsycho.2006.06.009

Sakatani, K., Tanida, M., \& Katsuyama, M. (2010). Effects of aging on activity of the prefrontal cortex and autonomic nervous system during mental stress task. Adv Exp Med Biol, 662, 473-478. doi:10.1007/978-1-4419-1241-1_68

Salmaso, D., \& Longoni, A. M. (1985). Problems in the assessment of hand preference. Cortex, 21(4), 533-549. doi:http://dx.doi.org/10.1016/S0010-9452(58)80003-9

Sanchez-Gonzalez, M. A., Guzik, P., May, R. W., Koutnik, A. P., Hughes, R., Muniz, S., . . Fincham, F. D. (2015). Trait anxiety mimics age-related cardiovascular autonomic modulation in young adults. J Hum Hypertens, 29(4), 274-280. doi:10.1038/jhh.2014.72

Segerstrom, S. C., \& Miller, G. E. (2004). Psychological stress and the human immune system: a meta-analytic study of 30 years of inquiry. Psychol Bull, 130(4), 601-630. doi:10.1037/0033-2909.130.4.601 
Seghier, M. L. (2008). Laterality index in functional MRI: methodological issues. Magn Reson Imaging, 26(5), 594-601. doi:10.1016/j.mri.2007.10.010

Shinba, T., Kariya, N., Matsui, Y., Ozawa, N., Matsuda, Y., \& Yamamoto, K. (2008). Decrease in heart rate variability response to task is related to anxiety and depressiveness in normal subjects. Psychiatry and clinical neurosciences, 62(5), 603-609. doi:http://dx.doi.org/10.1111/j.1440-1819.2008.01855.x

Spielberger, C. D., Gorsuch, R. L., Lushene, R., Vagg, P. R., \& Jacobs, G. A. (1983). Stait-Trait Anxiety Inventory (Form Y). Palo Alto, CA.

Tabachnick, B. G., \& Fidell, L. S. (2007). Using multivariate statistics (5th ed.). Boston, MA: Pearson Education, Inc.

Takizawa, R., Nishimura, Y., Yamasue, H., \& Kasai, K. (2014). Anxiety and performance: the disparate roles of prefrontal subregions under maintained psychological stress. Cereb Cortex, 24(7), 1858-1866. doi:10.1093/cercor/bht036

Tanida, M., Katsuyama, M., \& Sakatani, K. (2007). Relation between mental stress-induced prefrontal cortex activity and skin conditions: a near-infrared spectroscopy study. Brain Res, 1184, 210-216. doi:10.1016/j.brainres.2007.09.058

Tanida, M., Sakatani, K., Takano, R., \& Tagai, K. (2004). Relation between asymmetry of prefrontal cortex activities and the autonomic nervous system during a mental arithmetic task: near infrared spectroscopy study. Neurosci Lett, 369(1), 69-74. doi:10.1016/j.neulet.2004.07.076

Tarvainen, M. P., Niskanen, J. P., Lipponen, J. A., Ranta-Aho, P. O., \& Karjalainen, P. A. (2014). Kubios HRV--heart rate variability analysis software. Comput Methods Programs Biomed, 113(1), 210-220. doi:10.1016/j.cmpb.2013.07.024

Tarvainen, M. P., Ranta-Aho, P. O., \& Karjalainen, P. A. (2002). An advanced detrending method with application to HRV analysis. IEEE Trans Biomed Eng, 49(2), 172-175. doi:10.1109/10.979357

Task Force of the European Society of Cardiology. (1996). Heart rate variability standards of measurement, physiological interpretation, and clinical use. Eur Heart J, 17, 354-381. 
Thayer, J. F., Hansen, A. L., Saus-Rose, E., \& Johnsen, B. H. (2009). Heart rate variability, prefrontal neural function, and cognitive performance: the neurovisceral integration perspective on self-regulation, adaptation, and health. Ann Behav Med, 37(2), 141-153. doi:10.1007/s12160-009-9101-z

Thayer, J. F., \& Lane, R. D. (2000). A model of neurovisceral integration in emotion regulation and dysregulation. J Affect Disord, 61(3), 201-216. doi:http://dx.doi.org/10.1016/S0165-

\section{$\underline{0327(00) 00338-4}$}

Traina, M., Cataldo, A., Galullo, F., \& Russo, G. (2011). Effects of anxiety due to mental stress on heart rate variability in healthy subjects. Minerva Psichiatrica, 52(4), 227-231.

Ulrich-Lai, Y. M., \& Herman, J. P. (2009). Neural regulation of endocrine and autonomic stress responses. Nat Rev Neurosci, 10(6), 397-409. doi:10.1038/nrn2647

Wang, Y. P., \& Gorenstein, C. (2013). Psychometric properties of the Beck Depression InventoryII: a comprehensive review. Rev Bras Psiquiatr, 35(4), 416-431. doi:10.1590/1516-44462012-1048

Wittling, W., Block, A., Genzel, S., \& Schweiger, E. (1998). Hemisphere asymmetry in parasympathetic control of the heart. Neuropsychologia, 36(5), 461-468. doi:http://dx.doi.org/10.1016/S0028-3932(97)00129-2

Wittling, W., \& Genzel, S. (1995). Brain asymmetries in cerebral regulation of cortisol secretion. Homeostasis in Health \& Disease, 36, 1-5.

Woods, N. F., Lentz, M. J., Mitchell, E. S., \& Kogan, H. (1994). Arousal and stress response across the menstrual cycle in women with three perimenstrual symptom patterns. Res Nurs Health, 17(2), 99-110. doi:10.1002/nur.4770170205

World Medical Association. (2013). World medical association declaration of helsinki: Ethical principles for medical research involving human subjects. JAMA, 310(20), 2191-2194. doi:10.1001/jama.2013.281053

Yang, H., Wang, Y., Zhou, Z., Gong, H., Luo, Q., Wang, Y., \& Lu, Z. (2009). Sex differences in prefrontal hemodynamic response to mental arithmetic as assessed by near-infrared spectroscopy. Gender medicine, 6(4), 565-574. doi:http://dx.doi.org/10.1016/i.genm.2009.11.003 
Yu, B. H., Kang, E. H., Ziegler, M. G., Mills, P. J., \& Dimsdale, J. E. (2008). Mood states, sympathetic activity, and in vivo beta-adrenergic receptor function in a normal population. Depress Anxiety, 25(7), 559-564. doi:10.1002/da.20338

Zschucke, E., Renneberg, B., Dimeo, F., Wustenberg, T., \& Strohle, A. (2015). The stressbuffering effect of acute exercise: Evidence for HPA axis negative feedback. Psychoneuroendocrinology, 51, 414-425. doi:10.1016/j.psyneuen.2014.10.019 


\section{AUTHOR NOTES}

The present investigation was funded by the project Smart Aging (MIUR, Smart Cities Nazionale progetto n. 00442) and by ITALY® - Italian TALented Young ®esearchers Grant, University of Bergamo. 


\section{TABLES}

\section{Table 1}

\begin{tabular}{|l|c|c|}
\hline & Mean (SD) & Range (min - max) \\
\hline STAI-State & $34.56(7.06)$ & $24-52$ \\
\hline STAI-Trait & $41.37(9.79)$ & $23-65$ \\
\hline BDI & $8.23(6.71)$ & $0-32$ \\
\hline TE & $3.3(1.88)$ & $1-7$ \\
\hline SRQ & $14.55(5.57)$ & $5-28$ \\
\hline
\end{tabular}

Psychological questionnaire scores of all participants $(N=65)$ at baseline.

STAI = State Trait Anxiety Inventory; BDI = Beck Depression Inventory; TE = Task

Engagement; SRQ = Stress Rating Questionnaire. STAI-State scores were missing for 1 subject. 
Table 2

\begin{tabular}{|c|c|c|}
\hline & BDIsqrt & STAI-Trait \\
\hline BDlsqrt & 1 & $.736^{\star *}$ \\
\hline STAI-Trait & $.736^{\star \star}$ & 1 \\
\hline STAI-State & $.29^{\star}$ & $.405^{\star *}$ \\
\hline HR (B) & $-.367^{\star *}$ & -.198 \\
\hline HR (C) & $-.318^{*}$ & -.148 \\
\hline HR (E) & $-.304^{*}$ & -.168 \\
\hline logHFpow (B) & $.316^{*}$ & $.273^{*}$ \\
\hline logHFpow (C) & $.333^{* *}$ & $.264^{*}$ \\
\hline logHFpow (E) & $.329^{*}$ & $.266^{*}$ \\
\hline
\end{tabular}

2-tailed Pearson correlation coefficients between psychological and cardiological indexes in all participants $(\mathrm{N}=65)$. BDlsqrt = Beck Depression Inventory (square-root transformation); STAI= State Trait Anxiety Inventory- Trait and State scales; HR = Heart Rate; logHFpow = High

Frequencies power $(\log 10$ transformed $) ;(B)=$ baseline; $(\mathrm{C})=$ control; $(\mathrm{E})=$ experimental. STAIState scores were missing for 1 subject.

* correlation is significant at .05 level

${ }^{\star *}$ correlation is significant at .001 level 


\section{FIGURES}

\section{Figure 1}

Graphical representation of experimental procedure.

\section{Figure 2}

On the left, International 10-20 System. Grey circles indicate the measurement areas (located over frontopolar and dorsolateral PFC). On the right, Grand mean of Oxy-Hb (black line) and deOxy-Hb (red line) concentration changes expressed in a.u. of 65 individuals in right $(\mathrm{CH} 1)$ and left $(\mathrm{CH} 2)$ channels during the entire procedure.

\section{Figure 3}

Delta changes in Stress Rating Questionnaire (SRQ) and Task Engagement (TE) in Control (C) and Experimental (E) conditions. Error bars indicate $\pm 95 \%$ confidence interval.

\section{Figure 4}

Delta changes in High Frequencies power of HRV (log10 transformed; panel a) and in Heart Rates (panel b) in Control (C) and Experimental (E) conditions. Error bars indicate $\pm 95 \%$ confidence interval. 


\section{Figure 1}

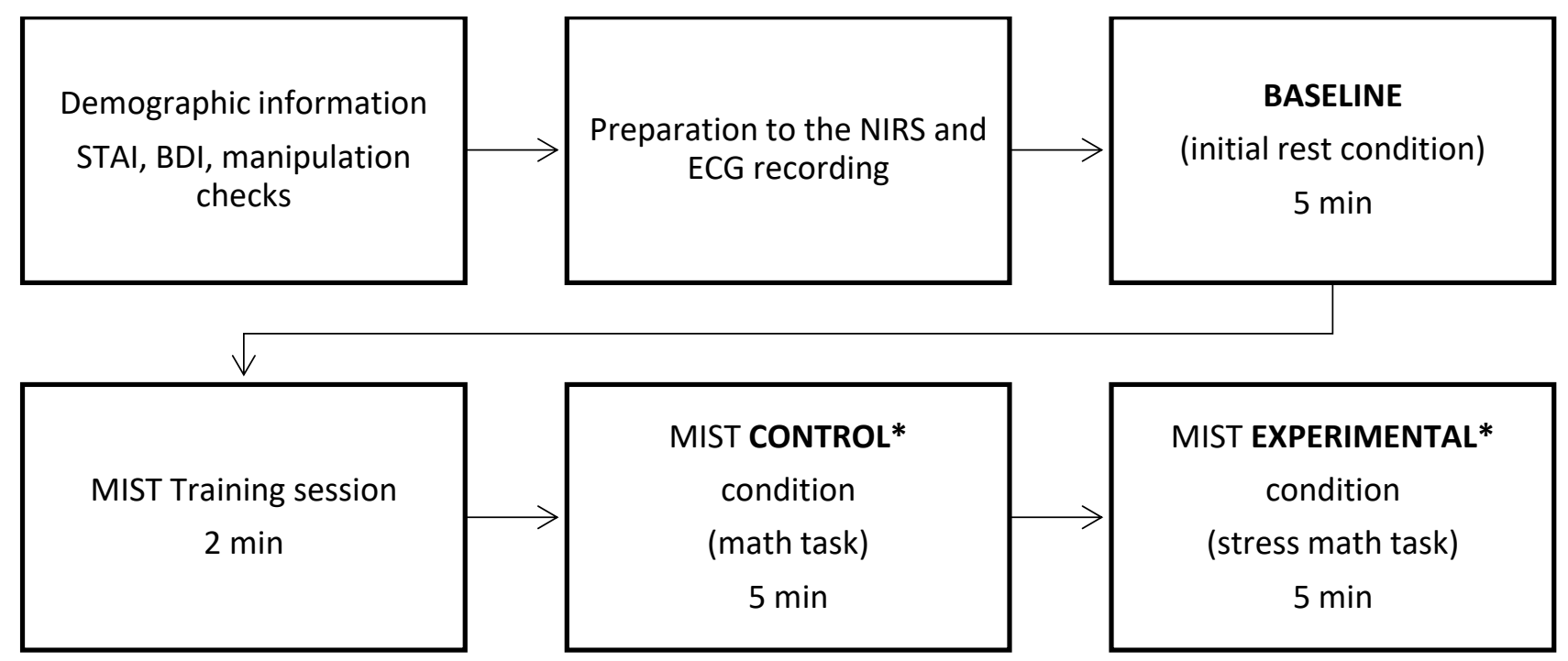

* Randomized

After each condition:

manipulation checks

After experimental: STAI-

State 


\section{Figure 2}
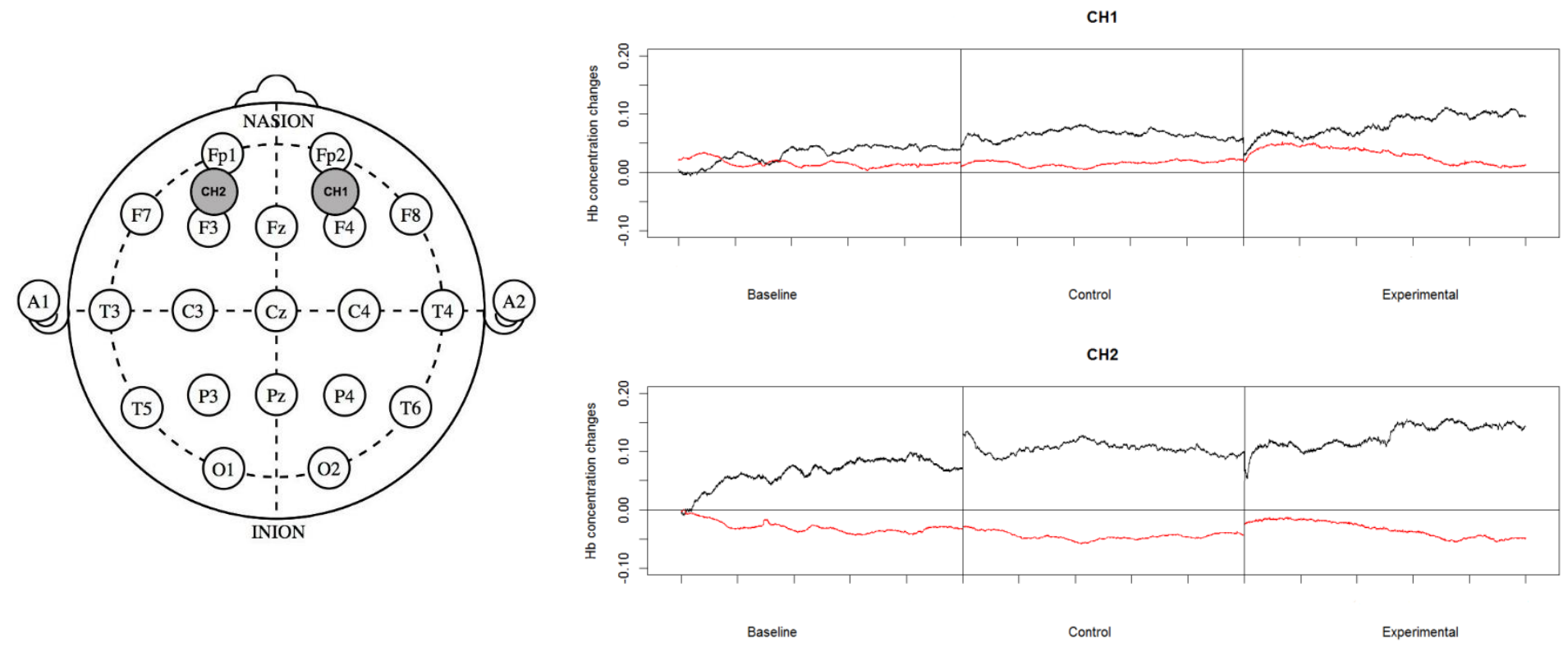
Figure 3

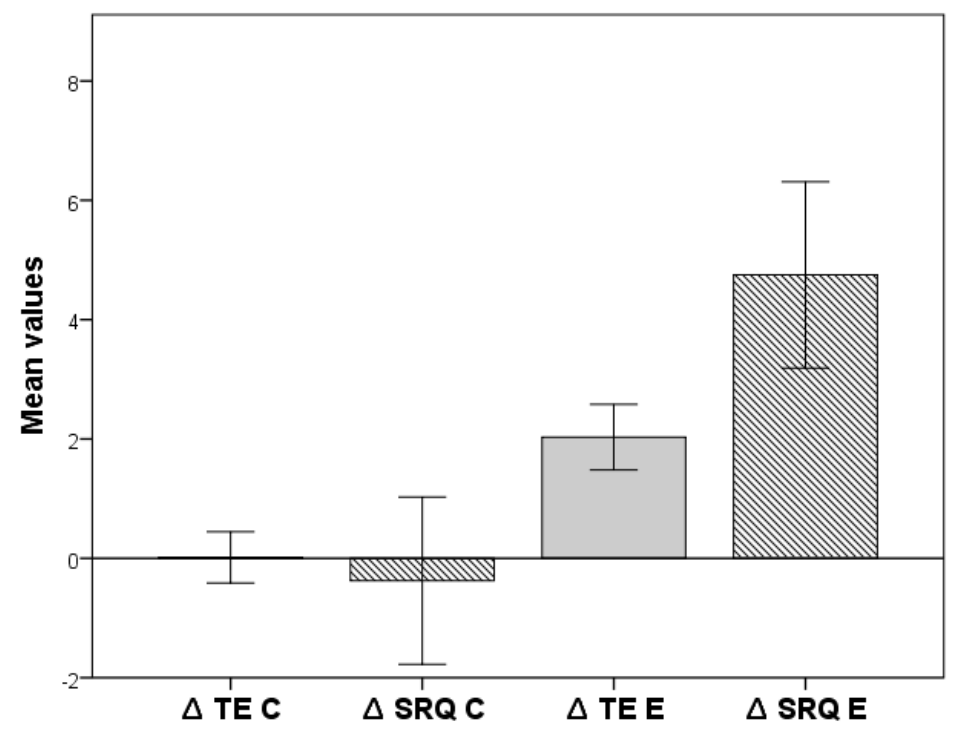


Stress, PFC, HRV and psychological distress 39

Figure 4

Panel a.

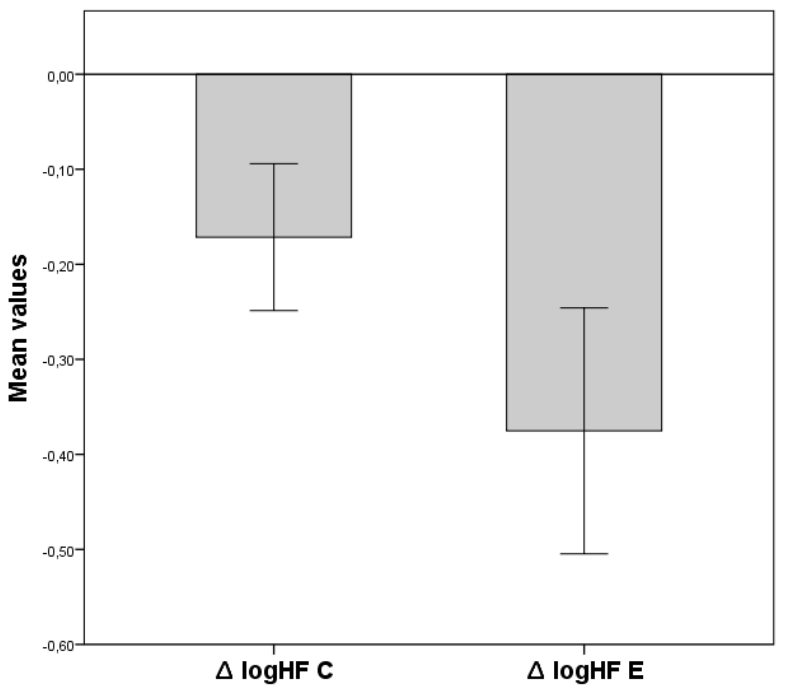

Panel b.

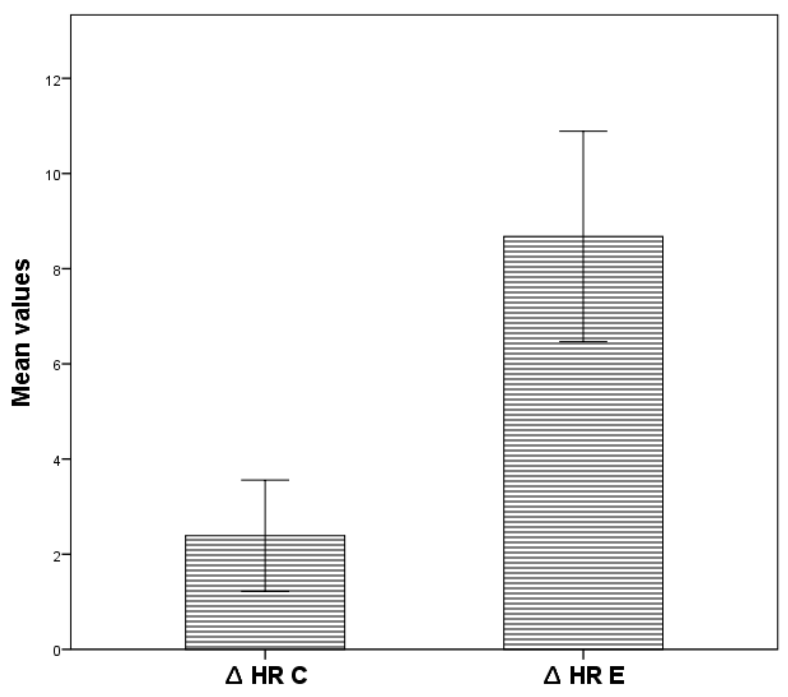

